\title{
The clone wars - revenge of the metastatic rogue state: the sarcoma paradigm
}

\author{
Holly L. Spraker 1,2*, Shawn L. Price ${ }^{2,3}$, Aashi Chaturvedi ${ }^{2,4}$, Joshua D. Schiffman ${ }^{1,2,4}$, Kevin B. Jones ${ }^{2,3}$, \\ Stephen L. Lessnick ${ }^{1,2,4}$, Mary Beckerle ${ }^{2,4}$ and R. Lor Randall ${ }^{2,3}$ \\ ${ }^{1}$ Division of Pediatric Hematology-Oncology, Primary Children's Medical Center, University of Utah, Salt Lake City, UT, USA \\ ${ }^{2}$ Center for Children's Cancer Research, Huntsman Cancer Institute, University of Utah, Salt Lake City, UT, USA \\ ${ }^{3}$ Department of Orthopedics, Sarcoma Services, Huntsman Cancer Institute, University of Utah, Salt Lake City, UT, USA \\ ${ }^{4}$ Department of Oncological Sciences, University of Utah, Salt Lake City, UT, USA
}

\section{Edited by:}

Douglas Hawkins, Seattle Children's

Hospital, USA

\section{Reviewed by:}

Ben Braun, University of California

San Francisco, USA

Scott C. Borinstein, Vanderbilt

University, USA

*Correspondence:

Holly L. Spraker, Division of Pediatric Hematology-Oncology, Primary

Children's Medical Center, 100 North Mario Capecchi Drive, Salt Lake City, UT 84113, USA.

e-mail: holly.spraker@imail.org
Ewing sarcoma (ES) is the second most common bone tumor affecting primarily adolescents and young adults. Despite recent advances in biological understanding, intensification of chemotherapeutic treatments, and progress in local control with surgery and/or radiation therapy, patients with metastatic or recurrent ES continue to have a dismal prognosis with less than $20 \%$ overall survival. All ES is likely metastatic at diagnosis although our methods of detection and classification may not account for this. Progressive disease may arise via a combination of: (1) selection of chemotherapy-resistant clones in primary tumor, (2) signaling from bone or lung microenvironments that may attract tumor cells to distant locations, and/or (3) genetic changes within the ES cells themselves due to DNAdamaging chemotherapeutic agents or other "hits." These possibilities and the evidence base to support them are explored.

Keywords: Ewing sarcoma, metastatic

\section{INTRODUCTION}

Ewing sarcoma (ES) is the second most common malignant bone tumor in adolescents and young adults, leading to more than 200 cases of cancer in the United States per year (SEER). Evidence of metastatic disease at diagnosis is the most clinically relevant prognostic factor, affecting 25\% of patients (Bernstein et al., 2006). Despite recent advances in biological understanding, intensification of chemotherapeutic treatments, and progress in local control with surgery and/or radiation therapy, patients with metastatic or recurrent ES continue to have a dismal prognosis with less than $20 \%$ overall survival.

Nearly every case of ES harbors a translocation of the EWSR1 gene, found on chromosome 22, with a member of the ETS family on chromosomes 11,21, or others. The classic ES translocation, $\mathrm{t}(11: 22)(\mathrm{q} 24 ; \mathrm{q} 12)$, creates the EWS-FLI1 fusion oncogene found in around $85 \%$ of ES tumors. Other fusion partners have also been described and account for the remaining 15\% (Turc-Carel et al., 1988; Delattre et al., 1992). The EWS-FLI1 oncogene influences the gene expression profile of tumor cells, directly or indirectly, driving aberrant expression of over 1000 genes (Smith et al., 2006). Interestingly, EWS-FLI1 expression is associated with activation of some genes and repression of others, illustrating the complexity of cellular response to this oncogenic transcription factor (May et al., 1993).

The ES translocation is thought to be the primary mechanism for tumorigenesis, but the heterogeneous biology found in the tumors of patients with ES suggests that additional molecular mechanisms are also involved (Toomey et al., 2010). Castillero-Trejo et al. (2005) using murine primary bone derived cells (mPBDC), have shown that serial passage of retrovirally transduced EWS-FLI1 mPBDCs produced tumors efficiently only in later-passage cells (> passage 15). In addition, Lessnick et al. (2002) established human primary fibroblast cell lines expressing the EWS-FLI1 fusion protein that underwent p53 mediated growth arrest showing that for tumor formation to proceed, there is likely a multistep process including the acquisition of other genetic changes (Lessnick et al., 2002). This research supports that the EWS-FLI1 translocation is an initiating event in sarcomagenesis, but that other biological processes are required for full tumorigenesis to occur (Lessnick et al., 2002; Castillero-Trejo et al., 2005).

Ewing sarcoma cells spread hematogenously to distant sites. Despite negative imaging studies and bone marrow biopsies, nearly all ES is likely micro-metastatic at diagnosis. Why then are clinically detectable metastases such an important prognostic factor? Perhaps tumor cells have differing potentials to grow and develop at distant sites determined by therapy selective pressures, microenvironmental signals, and changes that are intrinsic to the tumor cell's genes. These metastatic clones may undergo genetic changes that allow them to react differently to chemotherapeutic agents as well as signals in the microenvironment (of lung or bone) leading to a more aggressive, resistant phenotype. Many questions remain regarding the presence of ES clones that may lead to occult metastatic deposits and subsequently to recurrence of disease after completion of therapy. Are all ES metastatic clones similar or are they a heterogeneous population of rogues of varying clinical threat? This paper serves to review the current clinical knowledge about metastatic ES, to highlight current areas of research regarding the molecular pathways that influence ES metastasis, and to underscore questions that persist at this time. 
To survey the recently published literature in a comprehensive fashion, searches for "ES metastasis" and "metastatic ES" were performed via the PUBMED database. Results showed 586 articles that were published after 1999 on this topic. Abstracts were briefly scanned for relevance, and selected papers were reviewed in full. Original supporting data (some dated prior to 2000) were also used for the current review, based on the bibliography of the papers found.

\section{METASTATIC EWING SARCOMA: THE CLINICAL PERSPECTIVE}

Currently, metastatic disease is clinically defined by the presence of a ES specific translocation in the tissue biopsy of at least one tumor site (primary site) plus the presence of characteristic lesions (by imaging) in bones, lungs, or malignant cells identified in a staging bone marrow aspirate or biopsy. If diagnostic imaging is inconclusive for bone or lung sites, tissue biopsy may be undertaken to prove metastasis. Bone marrow is typically considered negative for metastatic disease if the cells present are morphologically hematopoietic in origin; peripheral blood is never clinically tested for ES cells. While these parameters clinically define metastatic disease and identify a high-risk sub-population of ES patients, they likely do not completely quantify a patient's burden of disease. Nor do such tests give us information about the genetic changes in a particular patient's ES tumor cells.

Approximately $25 \%$ of patients will present with metastatic disease, primarily with metastasis in the lungs, bony sites, and/or bone marrow. As with most cancers, the presence of metastasis is an important predictor of outcome. Patients with metastatic ES have a 5-year relapse free survival of $\sim 30 \%$ (versus localized disease $=61.3 \%, p<0.001$; Cotterill et al., 2000). Patients with metastatic disease have a statistically significant proportion of larger primary tumors $(>8 \mathrm{~cm})$ than patients without metastatic disease $(76.8$ versus $54.3 \%, p<0.00001)$ suggesting that primary tumor size may correlate with metastasis (Rodriguez-Galindo et al., 2008; Lee et al., 2010). On the other hand, some patients who present with small primary tumors do relapse with distant metastasis, indicating the propensity of ES for dissemination. In terms of outcomes, patients with primary pulmonary metastasis fare better than their counterparts with bone or bone marrow disease (5 year EFS 29-52\%) signifying that not all metastasis are equal (Paulussen et al., 1998).

The approach to treating those with metastatic disease involves systemic chemotherapy and local control of the primary site of disease. With regard to systemic therapy, there have been a variety of trials attempting to optimize a chemotherapeutic regimen specifically for patients with metastatic disease. These studies have investigated more intensive, time-compressed, and high-dose chemotherapy regimens, yet there has been minimal improvement in survival of patients with metastasis at presentation (Grier et al., 2003; Granowetter et al., 2009; Huang and Lucas, 2011) First-line agents are the same as for clinically localized disease (vincristine, doxorubicin, cyclophosphamide, ifosfamide, and etoposide), but there has been interest over the last decade in the use of camptothecin agents (such as topotecan and irinotecan) for metastatic disease (Wagner, 2011). The current front line Children's Oncology Group (COG) trial randomizes patients with localized ES to receive topotecan in combination with cyclophosphamide to attempt to improve survival rates, as this combination has shown promise in patients with relapsed or refractory disease.

Despite systemic chemotherapy and good local control modalities, even patients with clinically localized ES have a high-risk of relapse at distant sites, giving rise to the hypothesis that micrometastasis is almost universally present but undetected at initial diagnosis. Reports have shown that $25-30 \%$ of patients with clinically localized ES have detectable tumor cells in the peripheral blood or bone marrow by polymerase chain reaction (PCR; West et al., 1997). The question then arises as to whether these metastatic cells are clinically nefarious clones or rogues presenting a limited clinical threat. No data has been reported regarding detectable tumor cells at diagnosis for patients with metastatic disease, but one can hypothesize that it would be at least equivalent to if not higher than levels seen in localized ES. Further studies using peripheral blood PCR and/or flow cytometry in the metastatic population would be of interest as the nature of the clones in patients with clinically metastatic disease may differ from those that are detected peripherally in cases of clinically isolated disease. Perhaps comparative assessment of both primary and metastatic sites at diagnosis may also add to this body of knowledge. Other, smaller studies have suggested that detection of such EWS fusion oncogene transcripts in the blood or marrow after completion of therapy may portend relapse (Avigad et al., 2004). Again, are all metastatic clones of the same clinical threat?

Ewing sarcoma relapse usually presents within 5 years following intensive multi-agent chemotherapy and aggressive local control measures (Stahl et al., 2011). The predominant type of relapse in patients with initial metastatic disease is systemic (defined as distant recurrence only) with $73 \%$ of patients presenting almost evenly with pulmonary, bone, or multisystem recurrent sites (Stahl et al., 2011). The median survival time after a first recurrence is 9 months, and the 5-year overall survival is $12 \%$ for recurrent ES overall (Leavey et al., 2008). In recent data from the COG, the median time to recurrence in patients with metastatic disease at diagnosis is slightly earlier ( $\sim 1$ year; Leavey et al., 2008). Late recurrence, defined as greater than 2 years after initial diagnosis, is associated with a better prognosis (currently greater than $25 \%$ overall survival) whereas early recurrence portends a grave prognosis (OS =7-10\%; Huang and Lucas, 2011). Stahl et al. (2011) have corroborated that patients with initially clinically localized disease relapsed significantly later than those with metastatic disease at diagnosis (434 versus 563 days, $p<0.001$ ). In addition, the patients that relapsed earlier had a significantly worse outcome (OS $0-2$ years $=0.07,2-3$ years $=0.27,>3$ years $=0.30, p<0.001$; Stahl et al., 2011). Overall, the data suggests that metastatic patients are more likely to relapse early with systemic disease and have a worse prognosis. This leads to the hypothesis that either tumorrelated OR patient-related factors may lead to these ES clone's ability to evade destruction or removal during treatment. Disease that is cytoreduced into an initial state of subclinical tumor burden, but not fully eradicated, necessarily harbors resistant clones of ES. Chemotherapy provides both selective pressure favoring such clones as well as additional DNA damage able to induce genetic alterations that may generate new resistant clones. Early clinical recurrence implies that either the clones were resistant enough to 
grow during therapy or aggressive enough to rebound rapidly after therapy-suppression is lifted.

\section{THE TUMOR MICROENVIRONMENT}

The continued poor prognosis for patients with metastatic ES questions the possible need for distinctive treatment strategies designed specifically to prevent metastasis from the primary tumor site and to target and eradicate known or latent metastatic disease (Riggi and Stamenkovic, 2007). Research has focused both on understanding what triggers dissemination as well as what factors support tumor cell survival and proliferation in ectopic sites. ES cells have a propensity to metastasize to the lung, bone, and bone marrow (Arndt and Crist, 1999) suggesting an important role of tumor cell microenvironment that may direct this tissuespecific metastasis (Kerbel, 1995; Mundy, 2002). In recent years, several labs have focused their efforts on understanding the contribution of metastatic sites in attracting ES cells and supporting their growth. To prevent ES metastasis, an understanding of these unique tumor microenvironment and host-tumor interactions may offer new therapeutic targets.

Ewing sarcoma usually arises from bone, but can also metastasize to distant bones. The bone is a rich repository of growth factors including stem cell factor (SCF, the ligand for c-kit receptor), basic fibroblast growth factor (b-FGF), platelet derived growth factor (PDGF), and transforming growth factor- $\beta$ (TGF- $\beta$ ), therefore providing chemo-attractants for metastatic disease and a favorable environment for metastatic growth of many types of tumors (Mundy, 2002; Bussard et al., 2008). For example, metastatic sites highly express SCF in the bone marrow stromal cells (BMSCs), osteoblasts, and endothelial cells. Landuzzi et al. (2000) noted the presence of substantial surface c-kit receptors in six ES cell lines; transmembrane SCF was also found on five of the six cell lines. Exposure of these ES cell lines to exogenous SCF caused downregulation of its receptor, decreased chemoattraction of ES cell lines to SCF, and significantly reduced metastasis to lungs and other extra-pulmonary organs in a metastatic xenograft model. These data suggest that SCF expressed in potential metastatic sites may serve a chemoattractant role and that the c-kit receptor/SCF interaction is a potential target for decreasing ES metastasis (Landuzzi et al., 2000).

Fibroblast growth factor produced by BMSCs may promote a metastatic phenotype in ES tumors by increasing cell motility. Conditioned medium from BMSCs was shown to increase motility in human ES cell lines, specifically through activation of FGF receptor-1 (FGFR-1) by b-FGF and its downstream signaling cascade, phosphatidyl inositol 3 kinase (PI3K)-Rac-1 (Kamura et al., 2010). Receptors for FGF and PDGF are well-characterized and small molecule inhibitors against these catalytic receptors may provide a therapeutic option in ES.

Lyn, a member of the Src family of kinases, is a known regulator of tumor cell proliferation, adhesion, motility, and invasion. Targeting Lyn using a small interfering RNA (siRNA) or the small molecule inhibitor AP23994 resulted in suppression of tumor growth, decreased bony lysis due to tumor cells, and significantly fewer lung metastases in vivo (in athymic nude mice injected with TC71 human ES tumor cell lines; Guan et al., 2008). Here EWSFLI1 was shown to upregulate Lyn expression allowing increased bony lysis. Such lysis creates space for tumor growth, and provides easier access for tumor cells to the bone stroma where they may enter the circulation and metastasize to the lung. This data suggests that targeting Lyn upregulation may decrease the propensity of ES cells to metastasize.

Finally, ezrin, a membrane-cytoskeleton linking protein, has an effect on the development of metastasis in osteosarcoma and rhabdomyosarcoma (Khanna et al., 2004; Yu et al., 2004). Krishnan et al. (2006) have shown that this is true for ES as well. In normal cells, ezrin mediates signal transduction and cell to cell interactions, and promotes growth. Phosphorylation at threonine 567 (T567) of ezrin is essential for ezrin-mediated transduction of growth signals; mutation at this site, leading to absence of phosphorylation at T567 (so called ezrinT567A mutants), causes down-regulation of growth via the AKT/mTOR (mammalian target of rapamycin) pathway. Krishnan et al. (2006) have shown that ES cell lines (both previously established cell lines and primary cell lines from patient tumor samples) have high levels of ezrin expression, and ezrinT567A mutants maintain a significantly slower growth rate than those with wild type ezrin expression due to increased apoptosis. These data lend credence to ezrin's import in primary disease. In addition, injection of ezrinT567A mutant cells into mice produced fewer experimental metastases (2/30 mice developed metastasis) than mice injected with wild type ezrin (8/10 mice) showing that ezrin may also have a role in development and growth of metastasis in ES. Manipulation of the ezrin pathway may be explored in the future as a means to decrease the development of metastatic disease in ES (Krishnan et al., 2006).

\section{METASTATIC DISEASE, INSTABILITY OF THE CLONE}

As a tumor grows and metastasizes, its genome can continue to adapt allowing for creation of resistant clones that evade conventional therapies. Newer technologies, such as copy number analysis, rendering more detailed analysis of the genome than is possible with traditional cytogenetics, have been used to also look at genomic imbalances beyond translocation status in these tumors. As cancer genomes become more unstable, the number of copy number aberrations (CNA) increases (Jahromi et al., 2011). Copy number analysis of primary tumors may be a way to identify tumors that are at higher risk of metastasis or relapse than others. Several other tumor types have copy number changes that are being used to risk stratify therapy for patients (e.g., neuroblastoma; Attiyeh et al., 2005), but this is currently not possible for ES due to its rarity and challenge of obtaining a large enough cohort to study. Researchers continue to analyze genomic changes in ES samples (Armengol et al., 1997; Tarkkanen et al., 1999; Brisset et al., 2001; Ozaki et al., 2001; Savola et al., 2009), but the results have been discordant, possibly due to the small samples sizes used and/or the variable biology of the disease coupled with a lack of detailed clinical phenotyping.

In the late 1990 s to early 2000 s, several researchers described chromosomal gain in ES tumor samples - most commonly trisomy 1q [due to derivative chromosome (1:16)], trisomy 8 , and trisomy 12 in 25, 35, and 25\% of samples respectively (Armengol et al., 1997; Tarkkanen et al., 1999). The literature supports that $63-87 \%$ of ES tumor samples have CNAs detected by CGH 
(Armengol et al., 1997; Tarkkanen et al., 1999; Brisset et al., 2001; Ozaki et al., 2001; Savola et al., 2009). The most common of these CNAs have been analyzed for possible prognostic capacity, but the results have been varied. Many groups found no statistically significant changes in event-free survival or overall survival in ES based on CNAs (Armengol et al., 1997; Tarkkanen et al., 1999; Ozaki et al., 2001). However, two different groups found that the number of aberrations did significantly predict patient survival. Ozaki et al. (2001) showed in 48 patients that tumors with lower CNAs (less than five) were significantly associated with improved survival $(p=0.009)$. In addition, Savola et al. (2009) reported that tumors with higher numbers of CNA $(\geq 3)$ faired significantly worse in terms of OS $(p=0.030)$ and EFS $(p=0.049)$ than those with fewer CNAs. The findings to date are correlative in nature and require experimental testing to demonstrate whether CNA's reflect tumor age, behavioral attributes, and both. Currently, there is not a particular aberration or combination of changes being used to stratify patients for therapy.

The average number of CNAs in ES tumors varies in the literature. The most recent data using Agilent's $44 \mathrm{~K}$ oligoarray platform included 0-26 aberrations per tumor with a mean of 7.2 (Savola et al., 2009). Older reports, using various CGH techniques, reported on average two aberrations per tumor (range 1.14-3.6; Armengol et al., 1997; Tarkkanen et al., 1999; Brisset et al., 2001; Ozaki et al., 2001). Much of this variation may be due to the samples and techniques available for $\mathrm{CGH}$ at the time the data were collected. Mean copy number changes per tumor in other sarcomas such as osteosarcoma, malignant fibrous histiocytoma, and chondrosarcoma have been reported around 11, 6, and 6 respectively (Tarkkanen et al., 1995; Larramendy et al., 1997a,b). In ES, copy number changes were low in any given tumor sample lending credence to the primary translocation in ES being a single major genetic hit of great import in development and maintenance of tumors. This supports studies on cultured human ES cells in which knockdown of EWS-FLI expression was shown to be sufficient to reverse transformation and tumorigenic properties (May et al., 1993). As stated earlier, however, EWS-FLI1 expression is likely not the only genetic or epigenetic hit that is required for development and maintenance of ES (Lessnick et al., 2002; Castillero-Trejo et al., 2005). Hopefully, as the resolution of SNP microarrays increases and next-generation sequencing is introduced, more clinically relevant and cooperating CNAs may be discovered in ES.

Numbers of CNA in metastatic versus localized ES have been studied and have yielded inconclusive results. Brisset et al. (2001) found no difference between the type or average number of CNAs of primary tumor samples in patients who presented with metastasis. However, Savola et al. (2009) reported that metastatic tumors showed more CNA (mean 11.8) than primary tumors (mean 5.8). Finally, samples from relapsed tumors had greater than three times

\section{REFERENCES}

Armengol, G., Tarkkanen, M., Virolainen, M., Forus, A., Valle, J., Bohling, T., Asko-Seljavaara, S., Blomqvist, C., Elomaa, I., Karaharju, E., Kivioja, A. H., Siimes, M. A., Tukiainen, E., Caballín, M. R., Myklebost,
O., and Knuutila, S. (1997). Recurrent gains of $1 \mathrm{q}, 8$ and 12 in the Ewing family of tumours by comparative genomic hybridization. $\mathrm{Br}$. J. Cancer 75, 1403-1409.

Arndt, C. A., and Crist, W. M. (1999). Common musculoskeletal

as many copy number changes as did the samples of primary tumors ( 1.9 versus 4.4 per tumor sample; Armengol et al., 1997; Tarkkanen et al., 1999). From these data, it can be hypothesized that CNA appear with increased frequency as a tumor metastasizes or recurs, and that resistant clones with unstable genomes continue to gain genetic changes as such tumors progress. However, none of these studies have compared paired primary and metastatic lesion samples, so the clonal relationship of primary to metastatic tumors remains unknown in ES. Other tumor types, such as pancreatic carcinoma, have features in their metastatic deposits consistent with the parent clones, yet with the addition of newly acquired lesions reflecting that metastatic clones branched from the parent clone (Yachida et al., 2010). In patients with ES, the use of CNA on samples from both local and metastatic tumors may support such a mechanism and ultimately be an effective way to measure "fitness" of a clone for metastasis.

\section{CLOSING REMARIS}

Ewing sarcoma is an aggressive cancer of bone that targets the adolescent and young adult population. In its metastatic form, survival remains poor despite an influx of knowledge regarding tumor biology and intensification of therapy. Even if the disease is localized at diagnosis (and particularly if it is not), there are an unfortunate number of patients who will relapse; relapsed ES is very difficult to cure. Progressive disease is believed to arise via a combination of selection of chemotherapy-resistant clones, signaling from bone or lung microenvironments that may attract tumor cells to distant locations, and/or genetic changes within the ES cells themselves. Intrinsic genetic changes within the ES cells, due to a combination of therapy-related selection and DNA-damaging chemotherapeutic agents, may allow the attraction and migration of resistant ES clones to locations where they may thrive. As these clones compete to survive by evading chemotherapy and responding to signal from metastatic sites, enhanced genomic instability may favor their adaptation. While understanding the biology of the primary tumor is of utmost importance, it is also critical to understand genetic and epigenetic changes that are associated with the metastatic state in order to effectively treat sarcoma patients with systemic disease. Current advances in genetics and genomics provide new approaches to identify novel molecular markers that are associated with metastatic ES and define their clinical implications.

\section{ACKNOWLEDGMENTS}

Stephen L. Lessnick is supported by R01 CA140394, R21 CA138295, the Alex's Lemonade Stand Foundation, and the Terri Anna Perine Sarcoma Fund. Joshua D. Schiffman is supported by a SARC Career Development Award. R. Lor Randall is supported by the Terri Anna Perine Sarcoma Fund. M.C.B. is supported by R01 GM50877. The authors also acknowledge support from the Huntsman Cancer Foundation and P30CA042014.

tumors of childhood and adolescence. N. Engl. J. Med. 341, 342-352.

Attiyeh, E. F., London, W. B., Mosse, Y. P., Wang, Q., Winter, C., Khazi, D., McGrady, P. W., Seeger, R. C., Look, A. T., Shimada,
H., Brodeur, G. M., Cohn, S. L., Matthay, K. K., Maris, J. M., and Children's Oncology Group. (2005). Chromosome $1 p$ and 11q deletions and outcome in neuroblastoma. N. Engl. J. Med. 353, 2243-2253. 
Avigad, S., Cohen, I. J., Zilberstein, J., Liberzon, E., Goshen, Y., Ash, S., Meller, I., Kollender, Y., Issakov, J., Zaizov, R., and Yaniv, I. (2004). The predictive potential of molecular detection in the nonmetastatic Ewing family of tumors. Cancer 100, 1053-1058.

Bernstein, M., Kovar, H., Paulussen, M., Randall, R. L., Schuck, A., Teot, L. A., and Juergens, H. (2006). Ewing's sarcoma family of tumors: current management. Oncologist 11, 503-519.

Brisset, S., Schleiermacher, G., Peter, M., Mairal, A., Oberlin, O., Delattre, O., and Aurias, A. (2001). CGH analysis of secondary genetic changes in Ewing tumors: correlation with metastatic disease in a series of 43 cases. Cancer Genet. Cytogenet. 130, 57-61.

Bussard, K. M., Gay, C. V., and Mastro, A. M. (2008). The bone microenvironment in metastasis; what is special about bone? Cancer Metastasis Rev. 27, 41-55.

Castillero-Trejo, Y., Eliazer, S., Xiang, L., Richardson, J. A., and Ilaria, R. L. Jr. (2005). Expression of the EWS/FLI-1 oncogene in murine primary bone-derived cells results in EWS/FLI-1-dependent, Ewing sarcoma-like tumors. Cancer Res. 65, 8698-8705.

Cotterill, S. J., Ahrens, S., Paulussen, M., Jurgens, H. F., Voute, P. A., Gadner, H., and Craft, A. W. (2000). Prognostic factors in Ewing's tumor of bone: analysis of 975 patients from the European Intergroup Cooperative Ewing's Sarcoma Study Group. J. Clin. Oncol. 18, 3108-3114.

Delattre, O., Zucman, J., Plougastel, B., Desmaze, C., Melot, T., Peter, M., Kovar, H., Joubert, I., de Jong, P., Rouleau, G., Aurias, A., and Thomas, G. (1992). Gene fusion with an ETS DNA-binding domain caused by chromosome translocation in human tumours. Nature 359, 162-165.

Granowetter, L., Womer, R., Devidas, M., Krailo, M., Wang, C., Bernstein, M., Marina, N., Leavey, P., Gebhardt, M., Healey, J., Shamberger, R. C., Goorin, A., Miser, J., Meyer, J., Arndt, C. A., Sailer, S., Marcus, K., Perlman, E., Dickman, P., and Grier, H. E. (2009). Dose-intensified compared with standard chemotherapy for nonmetastatic Ewing sarcoma family of tumors: a Children's Oncology Group Study. J. Clin. Oncol. 27, 2536-2541.

Grier, H. E., Krailo, M. D., Tarbell, N. J., Link, M. P., Fryer, C. J., Pritchard, D. J., Gebhardt, M. C., Dickman,
P. S., Perlman, E. J., Meyers, P. A., Donaldson, S. S., Moore, S., Rausen, A. R., Vietti, T. J., and Miser, J. S.(2003). Addition of ifosfamide and etoposide to standard chemotherapy for Ewing's sarcoma and primitive neuroectodermal tumor of bone. $N$. Engl. J. Med. 348, 694-701.

Guan, H., Zhou, Z., Gallick, G. E., Jia, S. F., Morales, J., Sood, A. K., Corey, S. J., and Kleinerman, E. S. (2008) Targeting Lyn inhibits tumor growth and metastasis in Ewing's sarcoma. Mol. Cancer Ther. 7, 1807-1816.

Huang, M., and Lucas, K. (2011). Current therapeutic approaches in metastatic and recurrent Ewing sarcoma. Sarcoma. doi: 10.1155/ 2011/863210

Jahromi, M. S., Jones, K. B., and Schiffman, J. D. (2011). Copy number alterations and methylation in Ewing's sarcoma. Sarcoma 2011, 362173.

Kamura, S., Matsumoto, Y., Fukushi, J. I., Fujiwara, T., Iida, K., Okada, Y., and Iwamoto, Y. (2010). Basic fibroblast growth factor in the bone microenvironment enhances cell motility and invasion of Ewing's sarcoma family of tumours by activating the FGFR1-PI3K-Rac1 pathway. Br. J. Cancer 103, 370-381.

Kerbel, R. S. (1995). Significance of tumor-host interactions in cancer growth and metastases. Cancer Metastasis Rev. 14, 259-262.

Khanna, C., Wan, X., Bose, S., Cassaday, R., Olomu, O., Mendoza, A., Yeung, C., Gorlick, R., Hewitt, S. M., and Helman, L. J. (2004). The membrane-cytoskeleton linker ezrin is necessary for osteosarcoma metastasis. Nat. Med. 10, 182-186.

Krishnan, K., Bruce, B., Hewitt, S., Thomas, D., Khanna, C., and Helman, L. J. (2006). Ezrin mediates growth and survival in Ewing's sarcoma through the AKT/mTOR, but not the MAPK, signaling pathway. Clin. Exp. Metastasis 23, 227-236.

Landuzzi, L., De Giovanni, C., Nicoletti, G., Rossi, I., Ricci, C., Astolfi, A., Scopece, L., Scotlandi, K., Serra, M., Bagnara, G. P., Nanni, P., and Lollini, P. L. (2000). The metastatic ability of Ewing's sarcoma cells is modulated by stem cell factor and by its receptor c-kit. Am. J. Pathol. 157, 2123-2131. Larramendy, M. L., Tarkkanen, M., Blomqvist, C., Virolainen, M., Wiklund, T., Asko-Seljavaara, S., Elomaa, I., and Knuutila, S. (1997a). Comparative genomic hybridization of malignant fibrous histiocytoma reveals a novel prognostic marker. Am. J. Pathol. 151, 1153-1161.
Larramendy, M. L., Tarkkanen, M., Valle, J., Kivioja, A. H., Ervasti, H., Karaharju, E., Salmivalli, T., Elomaa, I., and Knuutila, S. (1997b). Gains, losses, and amplifications of DNA sequences evaluated by comparative genomic hybridization in chondrosarcomas. Am. J. Pathol. 150, 685-691.

Leavey, P. J., Mascarenhas, L., Marina, N., Chen, Z., Krailo, M., Miser, J., Brown, K., Tarbell, N., Bernstein, M. L., Granowetter, L., Gebhardt, M., Grier, H. E., and Children's Oncology Group. (2008). Prognostic factors for patients with Ewing sarcoma (EWS) at first recurrence following multi-modality therapy: a report from the Children's Oncology Group. Pediatr. Blood Cancer 51, 334-338.

Lee, J., Hoang, B. H., Ziogas, A., and Zell, J. A. (2010). Analysis of prognostic factors in Ewing sarcoma using a population-based cancer registry. Cancer 116, 1964-1973.

Lessnick, S. L., Dacwag, C. S., and Golub, T. R. (2002). The Ewing's sarcoma oncoprotein EWS/FLI induces a p53-dependent growth arrest in primary human fibroblasts. Cancer Cell 1, 393-401.

May, W. A., Lessnick, S. L., Braun, B. S., Klemsz, M., Lewis, B. C., Lunsford, L. B., Hromas, R., and Denny, C. T. (1993). The Ewing's sarcoma EWS/FLI-1 fusion gene encodes a more potent transcriptional activator and is a more powerful transforming gene than FLI-1. Mol. Cell. Biol. 13, 7393-7398.

Mundy, G. R. (2002). Metastasis to bone: causes, consequences and therapeutic opportunities. Nat. Rev. Cancer 2, 584-593.

Ozaki, T., Paulussen, M., Poremba, C., Brinkschmidt, C., Rerin, J., Ahrens, S., Hoffmann, C., Hillmann, A., Wai, D., Schaefer, K. L., Boecker, W., Juergens, H., Winkelmann, W., and Dockhorn-Dworniczak, B. (2001). Genetic imbalances revealed by comparative genomic hybridization in Ewing tumors. Genes Chromosomes Cancer 32, 164-171.

Paulussen, M., Ahrens, S., Burdach, S., Craft, A., Dockhorn-Dworniczak, B., Dunst, J., Frohlich, B., Winkelmann, W., Zoubek, A., and Jurgens, H. (1998). Primary metastatic (stage IV) Ewing tumor: survival analysis of 171 patients from the EICESS studies. European Intergroup Cooperative Ewing Sarcoma Studies. Ann. Oncol. 9, 275-281.

Riggi, N., and Stamenkovic, I. (2007) The biology of Ewing sarcoma. Cancer Lett. 254, 1-10.
Rodriguez-Galindo, C., Navid, F., Liu, T., Billups, C. A., Rao, B. N., and Krasin, M. J. (2008). Prognostic factors for local and distant control in Ewing sarcoma family of tumors. Ann. Oncol. 19, 814-820.

Savola, S., Klami, A., Tripathi, A., Niini, T., Serra, M., Picci, P., Kaski, S., Zambelli, D., Scotlandi, K., and Knuutila, S. (2009). Combined use of expression and CGH arrays pinpoints novel candidate genes in Ewing sarcoma family of tumors. BMC Cancer 9, 17. doi:10.1186/1471-2407-9-17

Smith, R., Owen, L. A., Trem, D. J., Wong, J. S., Whangbo, J. S., Golub, T. R., and Lessnick, S. L. (2006) Expression profiling of EWS/FLI identifies NKX2.2 as a critical target gene in Ewing's sarcoma. Cancer Cell 9, 405-416.

Stahl, M., Ranft, A., Paulussen, M., Bolling, T., Vieth, V., Bielack, S., Gortitz, I., Braun-Munzinger, G., Hardes, J., Jurgens, H., and Dirksen, U. (2011). Risk of recurrence and survival after relapse in patients with Ewing sarcoma. Pediatr. Blood Cancer 57, 549-553.

Tarkkanen, M., Karhu, R., Kallioniemi, A., Elomaa, I., Kivioja, A. H., Nevalainen, J., Bohling, T., Karaharju, E., Hyytinen, E., Knuutila, S., and Kallioniemi, O.-P. (1995). Gains and losses of DNA sequences in osteosarcomas by comparative genomic hybridization. Cancer Res. 55, 1334-1338.

Tarkkanen, M., Kiuru-Kuhlefelt, S., Blomqvist, C., Armengol, G., Bohling, T., Ekfors, T., Virolainen, M., Lindholm, P., Monge, O., Picci, P., Knuutila, S., and Elomaa, I. (1999). Clinical correlations of genetic changes by comparative genomic hybridization in Ewing sarcoma and related tumors. Cancer Genet. Cytogenet. 114, 35-41.

Toomey, E. C., Schiffman, J. D., and Lessnick, S. L. (2010). Recent advances in the molecular pathogenesis of Ewing's sarcoma. Oncogene 29, 4504-4516.

Turc-Carel, C., Aurias, A., Mugneret, F., Lizard, S., Sidaner, I., Volk, C., Thiery, J. P., Olschwang, S., Philip, I., Berger, M. P., Philip, T., Lenoir, G. M., and Mazabraud, A. (1988). Chromosomes in Ewing's sarcoma. I. An evaluation of 85 cases of remarkable consistency of $\mathrm{t}(11 ; 22)(\mathrm{q} 24 ; \mathrm{q} 12)$. Cancer Genet. Cytogenet. 32, 229-238.

Wagner, L. (2011). Camptothecin-based regimens for treatment of Ewing sarcoma: past studies, and future directions. Sarcoma 2011,957957. 
West, D. C., Grier, H. E., Swallow, M. M., Demetri, G. D., Granowetter, L., and Sklar, J. (1997). Detection of circulating tumor cells in patients with Ewing's sarcoma and peripheral primitive neuroectodermal tumor. J. Clin. Oncol. 15, 583-588.

Yachida, S., Jones, S., Bozic, I., Antal, T., Leary, R., Fu, B., Kamiyama, M., Hruban, R. H., Eshleman, J. R., Nowak, M. A., Velculescu, V. E., Kinzler, K. W., Vogelstein, B., and Iacobuzio-Donahue, C. A. (2010). Distant metastasis occurs late during the genetic evolution of pancreatic cancer. Nature 467, 1114-1117.

Yu, Y., Khan, J., Khanna, C., Helman, L.,'Meltzer, P. S., and Merlino, G. (2004). Expression profiling identifies the cytoskeletal organizer ezrin and the developmental homeoprotein Six-1 as key metastatic regulators. Nat. Med. 10, 175-181.

Conflict of Interest Statement: The authors declare that the research was conducted in the absence of any commercial or financial relationships that could be construed as a potential conflict of interest.

Received: 27 July 2011; paper pending published: 25 August 2011; accepted: 03 January 2012; published online: 16 January 2012.

Citation: Spraker HL, Price SL, Chaturvedi A, Schiffman JD, Jones KB, Lessnick SL, Beckerle $M$ and Randall $R L$ (2012) The clone wars - revenge of the metastatic rogue state: the sarcoma paradigm. Front. Oncol. 2:2. doi: 10.3389/fonc.2012.00002

This article was submitted to Frontiers in Pediatric Oncology, a specialty of Frontiers in Oncology.

Copyright (c) 2012 Spraker, Price, Chaturvedi, Schiffman, Jones, Lessnick, Beckerle and Randall. This is an openaccess article distributed under the terms of the Creative Commons Attribution Non Commercial License, which permits non-commercial use, distribution, and reproduction in other forums, provided the original authors and source are credited. 